\title{
Clinical Study \\ Determinants of Catch-Up Growth in International Adoptees from Eastern Europe
}

\author{
Bradley S. Miller, ${ }^{1}$ Maria G. Kroupina, ${ }^{1,2,3}$ Patrick Mason, ${ }^{4}$ Sandra L. Iverson, ${ }^{1,2}$ \\ Christine Narad, ${ }^{4}$ John H. Himes, ${ }^{5}$ Dana E. Johnson, ${ }^{1,2,3}$ and Anna Petryk ${ }^{1,6}$
}

${ }^{1}$ Department of Pediatrics, University of Minnesota, Minneapolis, MN 55455, USA

${ }^{2}$ International Adoption Medicine, University of Minnesota, Minneapolis, MN 55455, USA

${ }^{3}$ Center for Neurobehavioral Development, University of Minnesota, Minneapolis, MN 55455, USA

${ }^{4}$ International Adoption Center, Inova Fairfax Hospital for Children, Fairfax, VA 22031, USA

${ }^{5}$ Department of Epidemiology, University of Minnesota, Minneapolis, MN 55455, USA

${ }^{6}$ Division of Endocrinology, University of Minnesota Children's Hospital, MMC 8404,

13-124 Phillips-Wangensteen Building, SE Minneapolis, MN 55455, USA

Correspondence should be addressed to Anna Petryk, petry005@umn.edu

Received 13 October 2010; Accepted 23 November 2010

Academic Editor: P. Cohen

Copyright () 2010 Bradley S. Miller et al. This is an open access article distributed under the Creative Commons Attribution License, which permits unrestricted use, distribution, and reproduction in any medium, provided the original work is properly cited.

Children raised in orphanages frequently experience growth suppression due to multiple risk factors. Placing such children in more nurturing environments through adoption leads to significant catch-up growth (CUG), the determinants of which are not entirely understood. The goal of this study was to perform an auxological evaluation and examine the degree and correlates of CUG in international adoptees. Children adopted from Eastern Europe, ( $n=148,71$ males), 7 to 59 months of age, were recruited within 3 weeks of their arrival to the US. At baseline, mean height SDS was $-1.2 \pm 1.1$ and $22 \%$ were $<-2$ SDS for height. IGF1 and/or IGFBP-3 levels $<-2$ SDS were present in 32\%. CUG, defined as a gain of $>+0.5$ in height SDS, was seen in $62 \%$ of adoptees at 6 months after adoption; 7\% of children remained $<-2$ SDS for height (two had growth hormone deficiency). Growth factors improved in the majority of children. Younger age, greater degree of initial growth failure, and higher caloric intake were significantly associated with improved linear growth in multiple regression models. In summary, most adoptees demonstrate excellent CUG within six months after adoption. If growth failure persists after 6 months of appropriate caloric intake, nutritionindependent causes should be considered.

\section{Introduction}

Since 2000, over 160,000 children have come to the United States as international adoptees [1]. More than 25\% of these children have come from Eastern European countries having received primarily institutional care. Children in these orphanages frequently endure significant social deprivation that may impact their physical growth due to suppression of hypothalamic function with consequent low levels of growth factors [2]. We and others have previously documented significant physical growth delays in up to $34 \%$ of international adoptees from Eastern Europe, South America, and Asia [35]. Of the many hereditary, environmental, and hormonal factors that may contribute, we have previously shown that age, prenatal factors (birth weight and risk of fetal alcohol syndrome), and growth factors (Insulin-like Growth Factor Binding Protein-3, IGFBP-3) are independently associated with the degree of growth delay in adoptees upon arrival into the US [4].

A rapid period of catch-up growth (CUG) is seen following a variety of growth delays when the causative conditions improve. The Growth Hormone-(GH-)Insulinlike Growth Factor (IGF) system is known to be involved in CUG following many causes of growth delay, but limited data are available on the role of GH-IGF system in CUG following adoption [6]. The goal of this prospective longitudinal 
TABLE 1: Characteristics of international adoptees upon arrival to USA and after 6 months.

\begin{tabular}{|c|c|c|c|c|c|c|}
\hline & \multicolumn{3}{|c|}{ Arrival } & \multicolumn{3}{|c|}{6 months } \\
\hline & $n$ & Mean $\pm S D$ & $\%<-2$ SDS & $n$ & Mean \pm SD & $\%<-2$ SDS \\
\hline Age & 148 & $20.1 \pm 9.76$ & $\mathrm{~N} / \mathrm{A}$ & 132 & $26.4 \pm 10.2$ & N/A \\
\hline Gender & 148 & 77F 71M & $48.0^{\mathrm{c}}$ & 132 & $68 \mathrm{~F} 64 \mathrm{M}$ & $48.5^{\mathrm{c}}$ \\
\hline Height SDS & 148 & $-1.22 \pm 1.07$ & 21.6 & 132 & $-0.58 \pm 0.99 *$ & $6.8^{*}$ \\
\hline Weight SDS & 148 & $-1.44 \pm 1.30$ & 33.8 & 132 & $-0.62 \pm 1.13^{*}$ & $9.8^{*}$ \\
\hline Wt for Ht SDS & 148 & $-0.45 \pm 1.24$ & 9.5 & 132 & $-0.10 \pm 1.14^{*}$ & $4.5^{*}$ \\
\hline OFC SDS & 148 & $-0.65 \pm 1.13$ & 12.2 & 130 & $-0.26 \pm 1.24^{*}$ & $8.5^{*}$ \\
\hline IGF-1 SDS & 133 & $-1.13 \pm 1.42$ & 27.8 & 124 & $-0.95 \pm 1.34$ & 22.6 \\
\hline BP-3 SDS & 136 & $-0.92 \pm 1.10$ & 12.5 & 124 & $-0.52 \pm 1.03^{*}$ & $5.6^{*}$ \\
\hline Dietary intake & 115 & $108 \pm 33$ & N/A & 111 & $125 \pm 34^{*}$ & $\mathrm{~N} / \mathrm{A}$ \\
\hline$\% \mathrm{DRI}^{\mathrm{b}}$ & 102 & $135 \pm 42$ & N/A & 103 & $157 \pm 42^{*}$ & $\mathrm{~N} / \mathrm{A}$ \\
\hline BW (kg) & 104 & $2.75 \pm 0.71$ & $32.7^{\mathrm{d}}$ & & & \\
\hline
\end{tabular}

${ }^{\mathrm{a}} \mathrm{Kcal} / \mathrm{kg}$; ${ }^{\mathrm{b}} \%$ Dietary reference Intake; ${ }^{\mathrm{c}}$ Percent male; ${ }^{\mathrm{d}} \mathrm{BW}<2.50 \mathrm{~kg} ;{ }^{*} P<.05$.

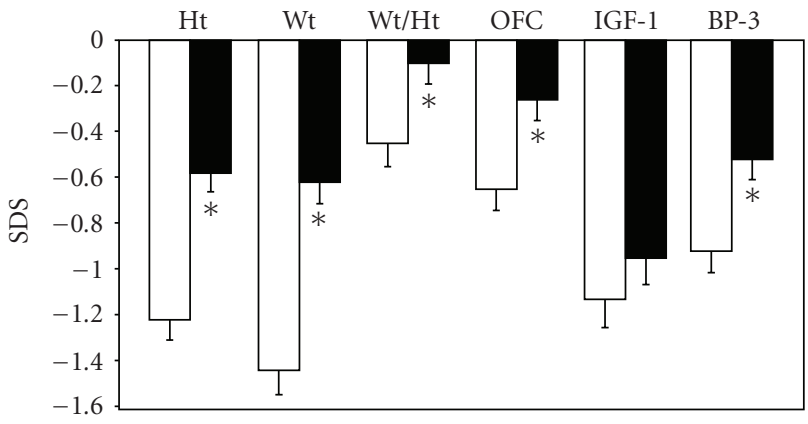

FIgURE 1: Characteristics of international adoptees upon arrival to USA and after 6 months. Standard Deviation Scores (SDSs) for height $(\mathrm{Ht})$, weight $(\mathrm{Wt})$, weight for height $(\mathrm{Wt} / \mathrm{Ht})$, occipitofrontal circumference (OFC), IGF-1, and BP-3 values at arrival (open bars) and after 6 months (shaded bars). ${ }^{*} P<.05$.

study was to examine the determinants of CUG in 148 postinstitutionalized international adoptees during the first 6 months after arrival into their adoptive families. We aimed to identify baseline factors that would help predict subsequent CUG and therefore help counsel the families, the degree of CUG, the usefulness of obtaining IGF-I and IGFBP-3 levels, and the impact of nutrition on CUG and the physiologic roles of nutrition and the growth hormone system on CUG.

\section{Patients and Methods}

2.1. Participants. Participants were part of a longitudinal study of growth of institutionalized children adopted into the USA from Eastern Europe (Russia, Kazakhstan, and Ukraine). The study was approved by the Institutional Review Boards of the University of Minnesota and Inova Fairfax Hospital for Children, and written informed consent was obtained from the adoptive parents. Participants who were between 6 and 59 months of age at adoption were recruited and enrolled through the International Adoption Clinics at the University of Minnesota and Inova Fairfax Hospital for Children between March 2004 and March 2007.
2.2. Study Design. Participants were examined within three weeks of arrival into the USA and then six months later. Auxological parameters included length or height (average of triplicate measurements), single determinations of weight, and occipitofrontal circumference (OFC). Midarm circumference, triceps, and subscapular skinfolds were also measured to assess the nutritional status of the adoptees in addition to liver function tests, total protein, and albumin, which are part of a routine pre-adoption evaluation. Preadoption historical data, including birth weight (BW) and time spent in institutions, such as hospitals and orphanages, were obtained via parent interview and translated pre-adoption medical records provided by the adoptive parents. Children were classified as being at high risk for fetal alcohol syndrome (FAS) if a moderate or severe facial phenotype was identified using facial photographic analysis software as previously described [7-10]. Dietary information was collected via a three-day diet record at each visit. Mothers recorded everything the child ate for three separate days in the two weeks prior to the clinic visit. The diet records were reviewed for completeness, and mothers were contacted afterward for additional clarification, if necessary. The diet records were entered into the Nutrition Data System for Research software (University of Minnesota) that generates intakes for 160 nutrients averaged over the three-day period of intake. Percentage of the US Dietary Reference Intakes (DRIs) were calculated using recommendations of the Institute of Medicine [11]. The DRI represents caloric requirements of a healthy child of a given age. If a child is receiving appropriate nutrition, the \%DRI is $100 \%$.

2.3. Biochemical Assays. Serum $(2.5 \mathrm{ml})$ was obtained through venipuncture and stored at $-20^{\circ} \mathrm{C}$ until shipping. IGF- 1 and IGFBP-3 were quantified by radioimmunoassay performed by Esoterix, Inc. (Calabasas, CA) and reported as actual values and Standard Deviation Scores (SDSs) [12]. TSH was measured by commercial chemiluminescent immunoassay as part of routine care. TSH was defined as high if it was above the normal range of the assay $(\geq 5.0 \mu \mathrm{IU} / \mathrm{mL})$. 


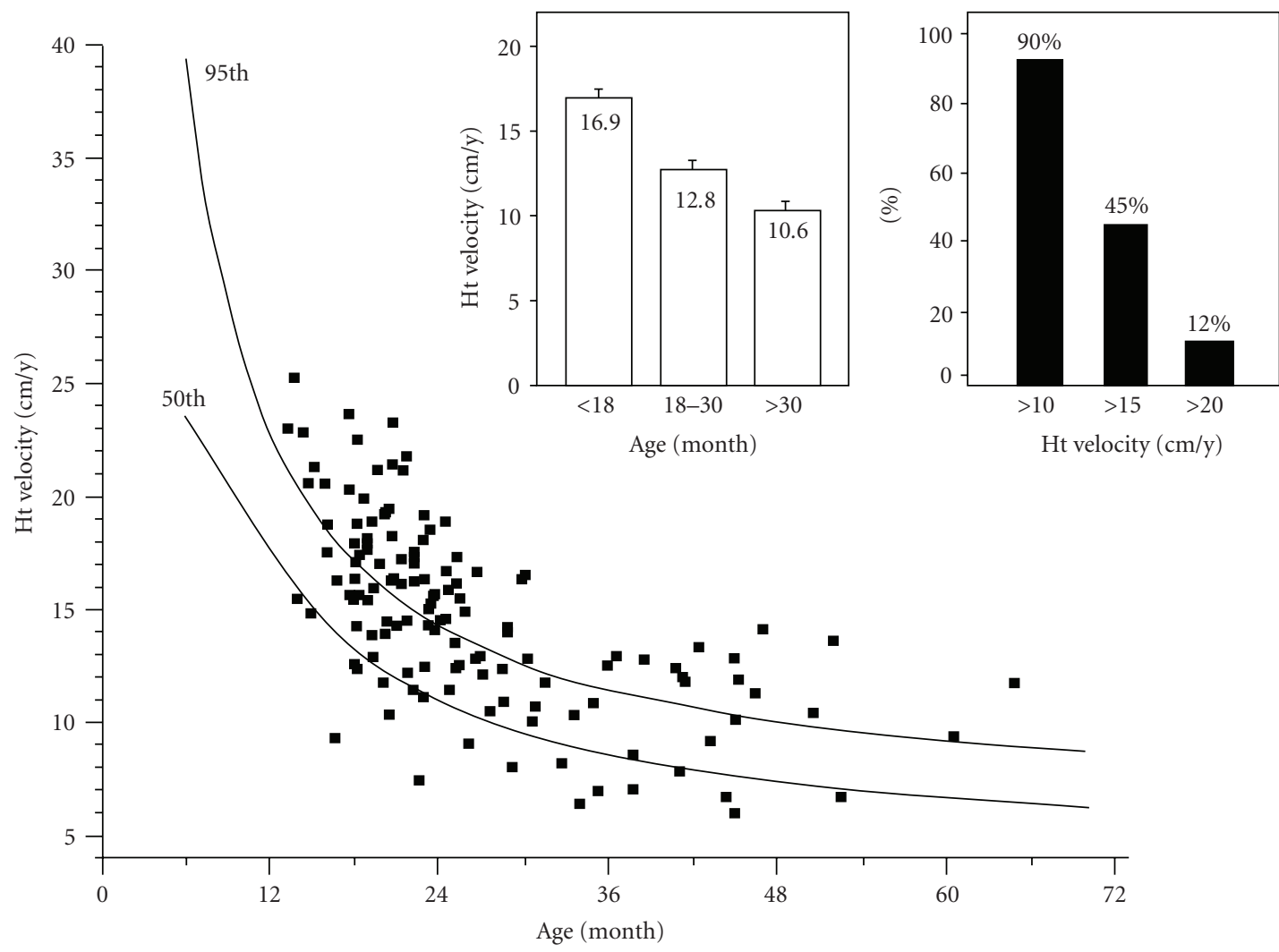

FIGURE 2: Age dependence of catch-up height velocity. Large panel: the height velocity $(\mathrm{cm} / \mathrm{y})$ at 6 months is plotted against age with normative reference data for 50th and 95th percentiles. Left inset: height velocity based upon age category. Right inset: percentage achieving height velocity $>10,15$, and $20 \mathrm{~cm} / \mathrm{y}$.

TABLE 2: Independent predictors of auxological change.

\begin{tabular}{|c|c|c|c|c|c|c|}
\hline \multirow[b]{2}{*}{ Variables at baseline } & \multicolumn{2}{|c|}{ Delta Ht SDS } & \multicolumn{2}{|c|}{ Delta Wt SDS } & \multicolumn{2}{|c|}{ Delta OFC SDS } \\
\hline & Coefficient & $P$ & Coefficient & $P$ & Coefficient & $P$ \\
\hline Age (months) & -0.014 & $.001^{*}$ & -0.005 & .574 & -0.006 & .653 \\
\hline Gender & 0.063 & .439 & -0.154 & .384 & 0.487 & .082 \\
\hline Height SDS & -0.143 & $.001^{*}$ & N/A & & 0.424 & $.017^{*}$ \\
\hline BP-3 SDS & -0.139 & .142 & -0.918 & $.038^{*}$ & N/A & \\
\hline IGF-1 SDS & -0.038 & .682 & N/A & & N/A & \\
\hline Dietary intake (Kcal/kg) & 0.300 & $.031^{*}$ & 0.009 & $.002 *$ & N/A & \\
\hline Weight SDS & N/A & & -0.478 & $.000^{*}$ & N/A & \\
\hline BW $(\mathrm{kg})$ & N/A & & 0.548 & $.000^{*}$ & N/A & \\
\hline OFC SDS & N/A & & N/A & & -0.407 & $.001^{*}$ \\
\hline
\end{tabular}

${ }^{*} P<.05$.

2.4. Data Analyses. Age- and gender-specific $Z$ scores for anthropometric variables were calculated based on the CDC 2000 Growth Charts using Epi Info 3.3 (Centers for Disease Control and Prevention, Atlanta, GA). For children older than 36 months, OFC SDS was estimated from the data of Roche et al. [13]. Age-specific percentiles for estimated annual height velocity were estimated from the figures and tables of Roche and Himes [14]. Statistical calculations and modeling were performed using SPSS software (SPSS, Inc., Chicago, IL). Analyses were conducted on all subjects that had complete data for that analysis, and the numbers of available cases are reported, accordingly. Changes in mean growth variables within the same individuals were evaluated using paired $t$-tests, and tests among multiple means for independent groups were by analysis of variance. Linear regression models for growth status and change were developed by considering only the subset of potential independent variables whose Spearman correlation coefficients with the dependent variables were statistically significant at $P \leq$ .10. Age and gender were included in all regression models irrespective of statistical significance. Partial regression coefficients were considered statistically significant at $P<.05$. 
Interactions among independent variables were investigated and rejected at $P>.10$. Adjusted odds ratios describing associations of tertiles of $\mathrm{Kcal} / \mathrm{kg}$, IGF-1, and IGFBP-3 with subsequent CUG were estimated from logistic regressions using CUG $(\geq 0.5 \mathrm{SD})$ as a dichotomous-dependent variable and adjusting for gender, age, and high risk for FAS status.

\section{Results}

The mean age at the time of initial evaluation was 20 months (range 7-59 months), which was on average $20 \pm$ 10 days after arrival into the USA (Table 1). The followup appointment was a mean of $6.05 \pm 0.86$ months later. As we have previously reported, international adoptees had significant growth failure upon arrival, which was more severe in children with low birth weight (LBW, <2500 g; $n=$ $34 / 104,32.7 \%)$ and high risk for FAS $(n=10 / 132,7.6 \%)$ [4]. All three growth parameters were affected; $22 \%$ of children were $<-2$ SDS for height, $34 \%$ were $<-2$ SDS for weight, and $12 \%$ were $<-2$ SDS for OFC. Ten percent of children were wasted at baseline using weight-for-height $<-2$ SDS. Low IGF-1 and/or IGFBP-3 levels (<-2 SDS) were present in 32\% (44 out of 136) at the initial visit. Liver function tests, total protein, and albumin were normal. At baseline, the mid-arm circumference was slightly below average when normalized for age $(-0.73$ SDS, $12.4 \%<-2$ SDS $)$ and height $(-0.56$ SDS, $10.8 \%<-2$ SDS), and the weight for height was near normal $(-0.45 \pm 1.24$ SDS with $9.5 \%$ below -2 SDS, Table 1$)$.

At 6 months, there was significant improvement in means for height SDS, weight SDS, OFC SDS, weight for height SDS, and IGFBP-3 SDS $(P<.05)$ (Table 1, Figure 1$)$. The change in IGF-I was not statistically significant. CUG, defined as a gain of $>+0.5$ in height SDS, was observed in $62 \%$ of children (82 out of 132), and 23\% (30 out of 132) gained $>+1$ height SDS over the six month period.

$84 \%$ of children had height velocity greater than the 50 th percentile for age with $55 \%$ greater than the 95 th percentile (Figure 2). $90 \%$ of children had height velocity $>10 \mathrm{~cm} / \mathrm{yr}$ and $12 \%>20 \mathrm{~cm} / \mathrm{yr}$. The degree of CUG was age dependent with mean growth velocities for children $<18$ months = $16.9 \pm 3.6 \mathrm{~cm} / \mathrm{yr}, 18-30$ months $=12.8 \pm 3.0 \mathrm{~cm} / \mathrm{yr}$, and $>30$ months $=10.6 \pm 2.5 \mathrm{~cm} / \mathrm{yr}(P<.001)$ (Figure 2$)$. Although the proportion of stunted children significantly decreased by 6 months, $6.8 \%$ of children $(N=132)$ remained below -2 SDS for height, $9.8 \%$ for weight, $8.5 \%$ for head circumference, and $4.5 \%$ for weight for height. Despite significant CUG in the group as a whole, IGF-1 and/or IGFBP-3 remained low in a significant number of children $(23 \%<-2$ SDS) $(29$ out of 124$)$.

In order to identify potential factors associated with CUG, we examined the relationships of the change in auxological parameters and growth factors with a number of environmental, historical, physical, medical, and hormonal variables. Young age, severity of short stature, and higher dietary intake at the initial visit were statistically significant independent predictors of improved growth (delta Ht SDS) (Table 2). Neither IGF-I nor IGFBP-3 SDS was an independent predictor of delta Ht SDS. Also, children in the lowest tertile of IGF-1 SDS were significantly more likely to show height CUG than those in the highest tertile ( $P=$ $.042, \mathrm{OR}=4.9,95 \% \mathrm{CI}: 1.1-22.9)$. Although associated with baseline growth failure, LBW and being at high risk for FAS were not significant predictors of CUG in height. However, low risk for FAS children was more likely to show height CUG than the high risk for FAS children $(P=.007, \mathrm{OR}=34.8,95 \% \mathrm{CI}: 2.7-457.6)$. Excluding high risk for FAS children from the regression analysis did not change the significant predictors. Mid-arm circumference and baseline weight for height were not independently predictive of baseline height SDS or degree of height CUG.

Because of the important role of nutrition in growth, we analyzed caloric intake at baseline and six months using a three-day diet record. Mean caloric intake was higher than the DRI at arrival $(\% \mathrm{DRI}=135 \pm 42 ; \mathrm{Kcal} / \mathrm{kg}=108 \pm 33)$ and was increased further at six months (\%DRI $=157 \pm 42$; $\mathrm{Kcal} / \mathrm{kg}=125 \pm 34 ; P<.001$ ) (Table 1). At baseline, $76 \%$ of children were consuming greater than the DRI (100\%) increasing to $93 \%$ at six months. Although mean height velocity was higher in younger children (Figure 2) and in those with higher caloric intake (Table 2), the mean increases in caloric intake per $\mathrm{kg}$ did not significantly differ across different ages $(P>.3$ by ANOVA) (Figure 3$)$.

Those children who had CUG (gain in height $>+0.5$ SDS) had statistically significantly higher caloric intake at both baseline and 6 months (Table 3 ) compared with other children. Caloric intake at 6 months was significantly correlated with subsequent change in height SDS $(r=0.318$, $P=.001)$. Children in the highest tertile of caloric intake $/ \mathrm{kg}$ at the initial visit were also significantly more likely to show height CUG than those in the lowest tertile of $\mathrm{Kcal} / \mathrm{kg}$ $(P=.033, \mathrm{OR}=4.2,95 \% \mathrm{CI}: 1.1-15.9)$. Relatively lower baseline weight SDS, higher birth weights, higher caloric intake, and lower baseline IGFBP-3 SDS were significant positive independent predictors of change in weight SDS (Table 2). Caloric intake at 6 months was also significantly correlated with the change in weight SDS $(r=0.441$, $P<.001)$ over the 6-month interval. OFC SDS and $\mathrm{Ht}$ SDS were independent predictors of growth in OFC, so that children with relatively smaller heads and greater heights at baseline had the most improvement in subsequent head growth (Table 2). If children who were high risk for FAS were excluded from the analysis, Ht SDS was no longer a significant independent predictor of subsequent OFC growth.

Nine children $(6.8 \%)$ remained stunted (height $<-2$ SDS) at the 6-month follow-up. Children who remained stunted were significantly older (mean 32 months, range 14 to 59 ), shorter (mean $-3.1 \mathrm{Ht}$ SDS, range -2.1 to -4.1 ), lighter (mean $-2.5 \mathrm{Wt}$ SDS, range -1.4 to -4.0 ) and had smaller heads (mean -1.66 OFC SDS, range -3.3 to 2 ) at the time of adoption than those who caught up (Table 4). There were no significant differences in mean baseline growth factors in those who remained stunted compared to those who caught up. Three of the children who had high risk for FAS remained stunted at 6 months. However, the degree of height CUG (delta Ht SDS) in high risk for FAS children 


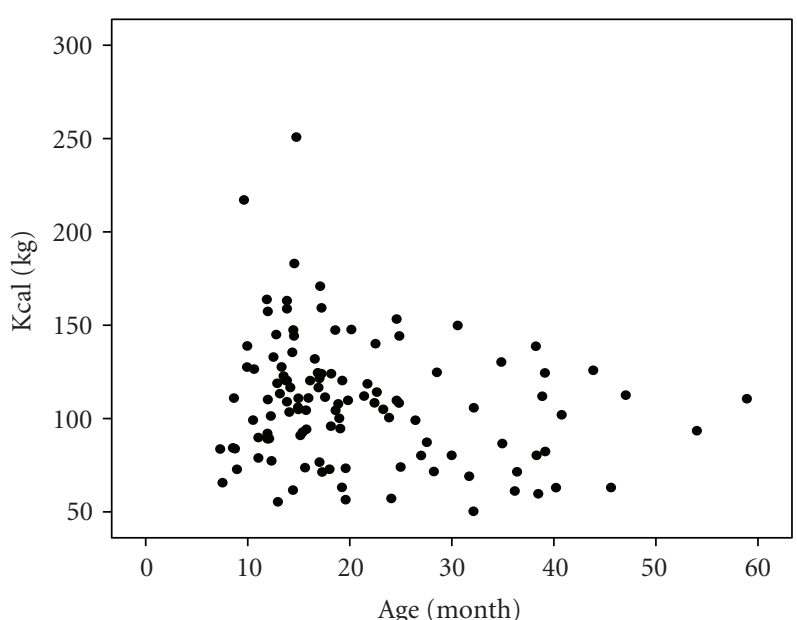

(a)

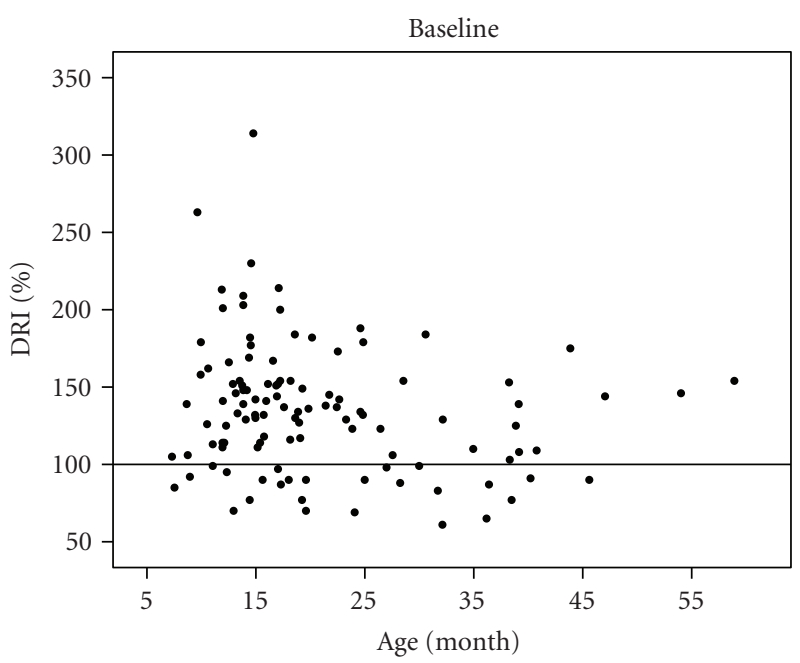

(c)

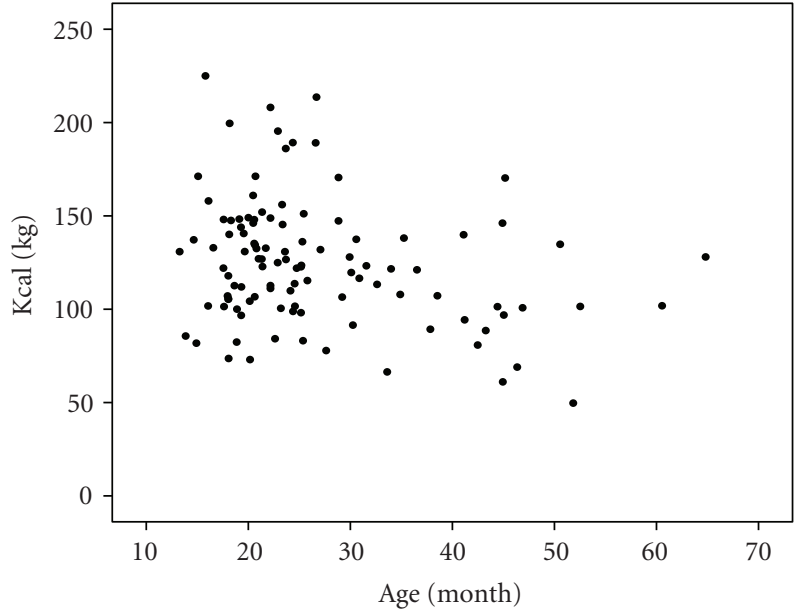

(b)

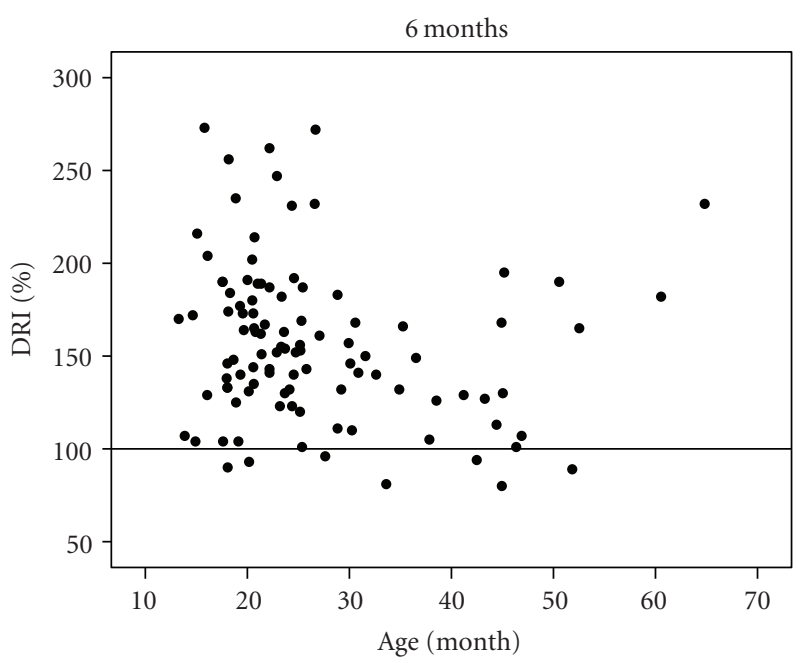

(d)

FIGURe 3: Dietary intake upon arrival to USA and after 6 months. Dietary intake for age expressed as kcal/kg (a and b) and \%DRI (c and d) at baseline ( $a$ and $c)$ and after 6 months (b and d). The \%DRI age reference (100\%) is indicated by a line (c and d).

TABLE 3: Dietary intake and catch-up growth.

\begin{tabular}{lccc}
\hline & $<+0.5$ Ht SDS $($ No CUG) & $>+0.5$ Ht SDS $(+$ CUG) & $P$ \\
\hline Baseline $(\mathrm{Kcal} / \mathrm{kg})$ & $98 \pm 30(n=36)$ & $114 \pm 34(n=68)$ & $.021^{*}$ \\
6 months $(\mathrm{Kcal} / \mathrm{kg})$ & $115 \pm 34(n=37)$ & $129 \pm 32(n=71)$ & $.039^{*}$ \\
Baseline $\left(\% \mathrm{DRI}^{\mathrm{a}}\right)$ & $122 \pm 38(n=35)$ & $142 \pm 43(n=67)$ & $.018^{*}$ \\
6 months $\left(\% \mathrm{DRI}^{\mathrm{a}}\right)$ & $143 \pm 46(n=33)$ & $163 \pm 39(n=70)$ &. $\mathbf{0 2 9}$ \\
\hline
\end{tabular}

a $\%$ Dietary reference intake; $* P<.05$.

was not statistically different from the larger cohort (data not shown).

Due to persistent short stature, children who did not catch-up by 6 months $(n=6)$ were referred for pediatric endocrinology evaluation. At the time of endocrine evaluation, five of these children had shown further CUG and were taller than $-2 \mathrm{Ht}$ SDS. Among the four children who remained stunted, two were identified by provocative growth hormone stimulation testing as having growth hormone deficiency. One female child with growth hormone deficiency, who was adopted at 14 months old and had associated optic nerve hypoplasia and FAS, has demonstrated an excellent response to growth hormone therapy with height at the 25th percentile at last evaluation five years after adoption. The pretreatment growth velocity was $6.8 \mathrm{~cm} /$ year (GV SD not established) compared to $12.3 \mathrm{~cm} /$ year $(+2.96$ SD) after 1 year of treatment with growth hormone. The other child a male who was 4 years old at the time of 
TABLE 4: Baseline characteristics of children who remained stunted versus those who caught up after 6 months.

\begin{tabular}{lccc}
\hline Baseline & Ht $<-2$ SDS & Ht $>-2$ SDS(caught-up) & $P$ \\
\hline Height SDS & $-3.10 \pm 0.69(n=9)$ & $-1.10 \pm 0.98(n=134)$ & $-1.37 \pm 1.31(n=134)$ \\
Weight SDS & $-2.53 \pm 0.81(n=9)$ & $-0.61 \pm 1.08(n=134)$ & $.000^{*}$ \\
OFC SDS & $-1.66 \pm 1.60(n=9)$ & $-1.10 \pm 1.40(n=121)$ & $.010^{*}$ \\
IGF-1 SDS & $-1.75 \pm 1.97(n=8)$ & $-0.89 \pm 1.08(n=124)$ & .216 \\
BP-3 SDS & $-1.13 \pm 1.48(n=8)$ & $19.3 \pm 9.1(n=134)$ & .529 \\
Age (months) & $32.0 \pm 13.7(n=9)$ & $109 \pm 34(n=105)$ & $.000^{*}$ \\
Dietary intake (Kcal/kg) & $109 \pm 23(n=9)$ & .975 \\
\hline
\end{tabular}

${ }^{*} P<.05$.

adoption with growth hormone deficiency was at low risk for FAS and also demonstrated a good response to growth hormone treatment. The pre-treatment growth velocity was $4.9 \mathrm{~cm} /$ year $(-0.82 \mathrm{SD})$ compared to $6.9 \mathrm{~cm} /$ year $(+2.12 \mathrm{SD})$ after 1 year of treatment with growth hormone. He is now growing along the 5 th percentile at last evaluation on growth hormone therapy five years after adoption.

Elevated TSH ( $>5 \mu \mathrm{IU} / \mathrm{ml}$, range 5.06-11.60) was seen in international adoptees $(n=18 / 110,16.4 \%)$ at baseline with a normal free T4 level. Of the 18 children with elevated TSH at baseline, 6 had normal TSH at the 6-month follow-up, 7 had no follow-up TSH reported, and 5 had elevated TSH at the end of the study. Children with elevated TSH at the end of the study ( $n=5 / 110,4.5 \%)$ were referred to a pediatric endocrinologist.

\section{Discussion}

In this prospective study of CUG in children following international adoption, we found that significant growth failure was present at baseline as published before by the authors [4]. During the first 6 months after adoption, these children experienced substantial CUG in length, weight, and OFC similar to previous cohorts $[15,16]$, and the degree of CUG positively correlated with the young age and the time of adoption as observed by others [5]. However, more than a third of children in this study did not show significant CUG ( $>+0.5$ SDS) during the 6-month followup period. $6.8 \%$ of children remained stunted $(<-2 \mathrm{Ht}$ SDS) at the end of the study, and two of these children were identified as having growth hormone deficiency. Older children with more severe baseline growth failure were more likely to remain stunted at follow-up. Although our previous analyses showed that LBW, high risk for FAS, and IGFBP-3 were significantly and independently associated with height SDS at the time of adoption [4], these variables were not significant independent predictors of subsequent height CUG, which in the case of IGFBP-3 was likely due to its correlation and colinearity with baseline Ht SDS [4]. Changes in IGF-1 and IGFBP-3 were positively correlated with linear growth, but were not independent predictors of growth restoration of adoptees. Therefore, it is likely that the improved nutrition and other changes, such as a more nurturing environment, have an important effect on the growth hormone axis influencing catch-up growth. Among the variables analyzed, only age, caloric intake (per kg), and baseline height were found to be independent predictors of height CUG.

Previous studies of CUG following growth failure caused by other mechanisms have shown improvement in serum levels of components of the GH-IGF system $[17,18]$. However, limited data are available on the role of $\mathrm{GH}$ IGF system in CUG following adoption [6]. We found that although baseline serum IGFBP-3 values correlated with the degree of linear growth failure [4] and IGF-1 and IGFBP3 SDS values improved in most children, neither IGFBP-3 nor IGF-1 was predictive of the degree of height CUG in the first six months following adoption. The finding that neither baseline growth factor levels nor the change in their values independently predicted the degree of CUG in height was unexpected. There are several potential explanations. It is possible that rapid changes in IGF-1 levels could have occurred between the time that these children were united with their adoptive parents and when the IGF1 values were measured in the clinic. This improvement could be related to improved nutrition or improved growth hormone secretion. Alternatively, low levels of IGFBP-3 may primarily reflect baseline growth hormone status in some patients. Improvement in IGFBP-3 over time may improve the delivery of IGF-1 to the target tissue. Finally, it is possible that other related factors, such as baseline height or caloric intake, are more important than these growth factors in this early, rapid CUG phase. Each of these possibilities requires further evaluation. These relationships suggest that although growth factors improve during this period, recovery from growth failure related to international adoption is a complex phenomenon that is dependent upon a number of factors and not solely related to restoration of normal function of the GH-IGF system per se.

Growth failure at the time of international adoption could represent a combination of malnutrition, reduced growth hormone secretion, and/or growth hormone resistance as observed in other conditions. For example, children who suffer from intrauterine growth retardation have been shown to have growth hormone resistance, but normal growth hormone production [19]. In psychosocial short stature, both growth hormone-responsive and growth hormone-resistant forms have been described [20]. Reversible suppression of growth hormone secretion may also occur in hyperphagic short stature, which is a condition 
associated with disordered eating behaviors (stealing food, hoarding food, foraging for discarded food) that are commonly seen in institutional settings $[21,22]$.

Access to sufficient macro- and micronutrients to support growth is critically important and, worldwide, the most common cause of growth failure during childhood. Nutritional demands vary depending on growth rates at different stages of development and any preexisting deficits due to pre- or postnatal malnutrition. During the most rapid growth phase between birth and 18 months the effects of even modest nutritional deficits may become magnified in children within institutional care settings. In this study, we found that $10 \%$ of the children were wasted (Wt $<-2$ SDS) on arrival that relative caloric intake was unusually high at baseline and substantially increased in most children. The degree of CUG for height and weight was related to calorie intake both at arrival and at six months. In addition, we have previously shown a high incidence of iron deficiency at adoption that persists at six months despite iron intake that is above the recommended daily allowance [23]. These findings emphasize the role of adequate nutrition support for CUG in international adoptees and highlight the need to monitor feeding issues that could impact nutritional intake.

\section{Conclusions}

We found that the growth hormone axis, as measured by IGF-1 and IGFBP-3, may be suppressed at baseline following international adoption. Growth factors improve during the 6-month interval after adoption in the majority of children. Baseline IGFBP-3 is an independent predictor of weight CUG, but not height CUG. Age and severity of growth failure at the time of adoption are independent predictors of the degree of subsequent CUG. LBW and high risk for FAS, associated with baseline growth failure, are not significant predictors of the degree of subsequent CUG, although a third of children who remained stunted after six months had facial features suggestive of FAS. Caloric intake is related to the degree of CUG for height and weight. As nutritional intervention appears to be extremely important during the first 6 months after adoption, we recommend that adoptive parents be made aware of the need for increased calories beyond typical age requirements. If growth failure persists after 6 months of appropriate caloric intake, nutritionindependent causes should be considered, for example, growth hormone deficiency. The older, the more severely stunted at the time of adoption, and those who are high risk for FAS should be monitored most closely.

\section{Acknowledgments}

This paper was funded in part by an investigator-initiated grant from the Genentech Center for Clinical Research in Endocrinology to A. Patryk, grants from the Viking Children's Fund and Minnesota Medical Foundation to D. E. Johnson, and from the National Center for Research Resources, National Institutes of Health to the General Clinical Research Center at the University of Minnesota
(M01-RR00400). The authors would like to thank the children and their adoptive parents for participating in this research study. They would also like to thank the clinical and research staff for their efforts.

\section{References}

[1] State USDo, "Immigrant Visas Issued to Orphans Coming to the US," Top Countries of Origin: FY2006, 2006.

[2] B. C. Gohlke, F. L. Frazer, and R. Stanhope, "Growth hormone secretion and long-term growth data in children with psychosocial short stature treated by different changes in environment," Journal of Pediatric Endocrinology and Metabolism, vol. 17, no. 4, pp. 637-643, 2004.

[3] D. E. Johnson, L. C. Miller, S. Iverson et al., "The health of children adopted from Romania," Journal of the American Medical Association, vol. 268, no. 24, pp. 3446-3451, 1992.

[4] B. S. Miller, M. G. Kroupina, S. L. Iverson et al., "Auxological evaluation and determinants of growth failure at the time of adoption in Eastern European adoptees," Journal of Pediatric Endocrinology and Metabolism, vol. 22, no. 1, pp. 31-39, 2009.

[5] M. H. van IJzendoorn, M. J. Bakermans-Kranenburg, and F. Juffer, "Plasticity of growth in height, weight, and head circumference: meta-analytic evidence of massive catch-up after international adoption," Journal of Developmental \& Behavioral Pediatrics, vol. 28, no. 4, pp. 334-343, 2007.

[6] M. B. Ranke, "Catch-up growth: new lessons for the clinician," Journal of Pediatric Endocrinology and Metabolism, vol. 15, no. 5, pp. 1257-1266, 2002.

[7] S. Astley, Introduction Manual: FAS Facial Photographic Analysis Software, FAS Diagnostic and Prevention Network, University of Washington, Seattle, Wash, USA, 2003.

[8] S. J. Astley and S. K. Clarren, "Diagnosing the full spectrum of fetal alcohol-exposed individuals: introducing the 4-digit diagnostic code," Alcohol \& Alcoholism, vol. 35, no. 4, pp. 400$410,2000$.

[9] S. J. Astley, J. Stachowiak, S. K. Clarren, and C. Clausen, "Application of the fetal alcohol syndrome facial photographic screening tool in a foster care population," Journal of Pediatrics, vol. 141, no. 5, pp. 712-717, 2002.

[10] L. C. Miller, W. Chan, A. Litvinova et al., "Fetal alcohol spectrum disorders in children residing in Russian orphanages: a phenotypic survey," Alcoholism: Clinical \& Experimental Research, vol. 30, no. 3, pp. 531-538, 2006.

[11] J. Otten, J. Hellwig, and L. Meters, Institute of Medicine Dietary Reference Intakes: The Essential Guide to Nutrient Requirements, National Academies Press, Washington, DC, USA, 2006.

[12] D. Buchwald, J. Umali, and M. Stene, "Insulin-like growth factor-I (somatomedin C) levels in chronic fatigue syndrome and fibromyalgia," Journal of Rheumatology, vol. 23, no. 4, pp. 739-742, 1996.

[13] A. F. Roche, D. Mukherjee, S. Guo, and W. M. Moore, "Head circumference reference data: birth to 18 years," Pediatrics, vol. 79, no. 5, pp. 706-712, 1987.

[14] A. F. Roche and J. H. Himes, "Incremental growth charts," American Journal of Clinical Nutrition, vol. 33, no. 9, pp. 20412052, 1980.

[15] D. E. Johnson, "Medical and developmental sequelae of early childhood institutionalizaiton in Eastern European adoptees," in The Effects of Early Adversity on Neurobehavioral Development: The Minnesota Symposia on Child Psychology, 
C. A. Nelson, Ed., pp. 113-162, Lawrence Erlbaum Associates, Mahwaw, NJ, USA, 2000.

[16] P. Mason and C. Narad, "Long-term growth and puberty concerns in international adoptees," Pediatric Clinics of North America, vol. 52, no. 5, pp. 1351-1368, 2005.

[17] B. Argüelles, V. Barrios, J. Pozo, M. T. Muñoz, and J. Argente, "Modifications of growth velocity and the insulin-like growth factor system in children with acute lymphoblastic leukemia: a longitudinal study," Journal of Clinical Endocrinology \& Metabolism, vol. 85, no. 11, pp. 4087-4092, 2000.

[18] M. Boguszewski, R. Bjarnason, C. Jansson, S. Rosberg, and K. Albertsson-Wikland, "Hormonal status of short children born small for gestational age," Acta Paediatrica Supplement, vol. 423, pp. 189-192, 1997.

[19] M. B. Ranke, A. Lindberg, C. T. Cowell et al., "Prediction of response to growth hormone treatment in short children born small for gestational age: analysis of data from KIGS (Pharmacia International Growth Database)," Journal of Clinical Endocrinology \& Metabolism, vol. 88, no. 1, pp. 125-131, 2003.

[20] R. M. Blizzard and A. Bulatovic, "Syndromes of psychosocial short stature," in Pediatric Endocrinology, F. Lifshitz, Ed., pp. 83-93, Marcel Dekker, New York, NY, USA, 3rd edition, 1996.

[21] J. Gilmour and D. Skuse, "A case-comparison study of the characteristics of children with a short stature syndrome induced by stress (Hyperphagic Short Stature) and a consecutive series of unaffected 'stressed' children," Journal of Child Psychology \& Psychiatry \& Allied Disciplines, vol. 40, no. 6, pp. 969-978, 1999.

[22] D. Skuse, A. Albanese, R. Stanhope, J. Gilmour, and L. Voss, "A new stress-related syndrome of growth failure and hyperphagia in children, associated with reversibility of growthhormone insufficiency," The Lancet, vol. 348, no. 9024, pp. 353-358, 1996.

[23] A. J. Fuglestad, A. E. Lehmann, M. G. Kroupina et al., "Iron deficiency in international adoptees from Eastern Europe," Journal of Pediatrics, vol. 153, no. 2, pp. 272-277, 2008. 